\title{
Fermentation quality of herbal tea residue and its application in fattening cattle under heat stress
}

\author{
Xiaona Zhuang ${ }^{\dagger}$, Zujing Chen ${ }^{\dagger}$, Xiaohong Sun ${ }^{\dagger}$, Fangjun Li, Junyi Luo, Ting Chen, Qianyun Xi, \\ Yongliang Zhang ${ }^{*}$ and Jiajie Sun ${ }^{*}$
}

\begin{abstract}
Background: Herbal tea residue (HTR) is generally considered to be the waste of herbal tea beverage production while it still retains rich nutrients and active substances. The main aim of the present study was to investigate the effect of fermentation technology on improving the quality of HTRs, and focus on the fermented HTR-induced alleviation of summer heat stress in fattening cattle.

Results: In this study, the waste HTR was fermented and then fed to a total of 45 fattening cattle that were divided into 3 groups (fermented HTR replaced 0, 15, 30\% of the forage component of the diet), and the feeding experiment was lasted for 40 days. The physiological indexes, growth performance and fecal microbiota of fattening cattle were evaluated and results showed that fermented HTR could effectively reduce the respiratory rate and rectal temperature of fattening cattle under heat stress, increase the daily feed intake and daily gain, and improve the antioxidant content and blood immune index. In addition, we studied the fecal microbiota composition of 6 fattening cattle in control and 30\% HTR substitution groups and found fermented HTR significantly changed the composition of fecal microbiota and increased microbial diversity, and correlation analysis suggested that the bacteria were closely related to fecal SCFA levels of fattening cattle under heat stress.
\end{abstract}

Conclusions: In this study, fermented HTR replaced $30 \%$ of the forage component of the diet that can change the intestine microorganisms, maintain health and alleviate the heat stress of fattening cattle.

Keywords: Herbal tea residue, Microorganism, Fattening cattle, Fermented feed, Heat stress

\section{Background}

Herbal medicine has a long history of being used to prevent and treat diseases in China [1]. Studies have shown that drinking herbal tea can help to relieve heat in the body and the symptoms of sore throat caused by summer

\footnotetext{
*Correspondence: jiajiesun@scau.edu.cn; jiajiesun@scau.edu.cn ${ }^{\dagger}$ Xiaona Zhuang, Zujing Chen and Xiaohong Sun contributed equally to this work.

Guangdong Provincial Key Laboratory of Animal Nutrition Control, Guangdong Engineering \& Research Center for Woody Fodder Plants, National Engineering Research Center for Breeding Swine Industry, Guangdong Laboratory for Lingnan Modern Agriculture, South China Agricultural University, Guangzhou 510642, Guangdong, China
}

heat stress [2]. Herbal tea drinks are consumed widely because of their natural ingredients, convenient drinking, and unique health benefits [3-5]. In some areas, such as Guangdong, China, drinking herbal tea has even become part of the culture [6]. More and more people are consuming herbal tea drinks, requiring large scale tea production and resulting in large amounts of HTR [7]. In the past, most of tea residues were regarded as waste and were burned or dumped into landfill [8], which was not only environmentally damaging, but also a represented a waste of resources. Fortunately, researchers are now paying attention to the resource utilization of tea residues. 
Researchers have found that tea residues can significantly improve the soil fertility, leading to their use as organic fertilizers [9]. In addition, tea residues have been developed as a non-conventional water adsorbent to remove water pollutants like methylene blue [10], antibiotics [11], and heavy metals, such as chromium [12], cadmium [13], copper [14], lead, zinc [15], and arsenic [16] from aqueous solutions.

Alternatively, tea residues can be used as an unconventional feed resource in animal production. Generally, during tea production, not all of the effective substances are completely dissolved, thus some tea residues still retain a good proportion of the nutrients and bioactive compounds [17]. Like tea residues, it is reported that HTR contains many residual bioactive substances, such as phenols, polysaccharides, organic acids, alkaloids, and essential oils, which have been proven to have anti-oxidant and anti-bacterial effects [18]. Moreover, tea residues also contain protein, carbohydrate, fat, fiber, vitamins, and minerals [18]. Previous studies have used tea residues as substitutes for antibiotics to help improve the gut microbial composition of grower pigs [19]. In addition, tea residues improved the abdominal fat accumulation of broiler chickens, and had a positive impact on egg yolk quality [20]. Besides, researchers demonstrated the potential of tea residues for feeding ruminants [17]. In another study, a fermented herbal residue was fed to Holstein heifers, which effectively promoted the growth and immune performance of cows under heat stress [21].

In our paper, the tested HTRs included Plumeria rubra flowers, Honeysuckle flowers, Chrysanthemum flowers, Mesona chinensis leaves, Prunella vulgaris leaves, Microcos paniculata leaves and Liquiritia glycyrrhiza roots. The potential feeding value of the HTRs prompted us to develop a suitable technology for animal production. Currently, to apply HTRs, problems of storage, unbalanced nutrition, and poor palatability need to be solved. Therefore, the main aim of the present study was to investigate the effect of fermentation technology on improving the quality of HTRs, and focus on the fermented HTR-induced alleviation of summer heat stress in fattening cattle.

\section{Results}

\section{Nutritional components and changes to the HTR} after fermentation

As shown in Table S1, the HTR contained a high water content $(75.10 \%)$ and the dry matter content was $24.90 \%$. As a proportion of the dry matter content, the crude protein, crude fat, and ash contents were 13.10, 2.60, $6.69 \%$, respectively. In addition, HTR also contained minerals, and the contents of potassium, calcium, magnesium, phosphorus, sulfur, and chlorine were $0.61,1.14$, $0.40,0.25,0.21$, and $0.51 \%$, respectively. The acid detergent fiber (ADF) and neutral detergent fiber (NDF) were 39.8 and 54.3\%; contents of lactate acid, acetic acid, and butyric acid were $4.67,1.58$, and $0.35 \%$, respectively; and the water soluble carbohydrate (WSC) was $3.10 \%$. The net energy for maintenance and net energy gain of HTR were 0.79 and $0.25 \mathrm{Mcal} / \mathrm{kg} \mathrm{DM}$, respectively.

As shown in Table 1, the $\mathrm{pH}$ value of the mixture of the HTR, oat hay, and bacteria decreased from 4.95 to 3.89 after fermentation. On the basis of dry matter, the crude protein in the HTR increased by $1.07 \%$ compared with that of the unfermented HTR. The contents of NDF and ADF decreased by 5.82 and $4.32 \%$ respectively. On the basis of dry matter, the contents of lactate, acetic acid, and propionic acid increased to 6.93, 0.62 and $112.33 \mathrm{~g} /$ $\mathrm{kg}$ DM, respectively. Basal diet composition and nutritional level of bovine feeding were shown in Table S2. Compared with $\mathrm{CN}$ group, LC group and $\mathrm{HC}$ group had more abundant crude protein $(11.25,11.32$ and $11.35 \%$, respectively). In addition, compared with $\mathrm{CN}$ group, LC group and $\mathrm{HC}$ group also had more abundant crude fat

Table 1 Nutrient composition of fermented herbal tea residue for 0 and 30 days

\begin{tabular}{lll}
\hline Item & Fermentation for 0 days & Fermentation for 30 days \\
\hline PH & $4.95 \pm 0.03$ & $3.89 \pm 0.04$ \\
Dry matter (\%) & $26.58 \pm 0.74$ & $24.94 \pm 0.51$ \\
Crude protein (\%) & $8.85 \pm 0.98$ & $9.92 \pm 0.82$ \\
Ash (\%) & $6.17 \pm 0.04$ & $6.56 \pm 0.06$ \\
Neutral detergent fiber (\%) & $60.11 \pm 1.30$ & $54.29 \pm 0.86$ \\
Acid detergent fiber (\%) & $34.33 \pm 0.28$ & $30.01 \pm 0.36$ \\
$\quad$ Acetic acid (g/kg) & $1.04 \pm 0.01$ & $6.93 \pm 0.06$ \\
$\quad$ Propionic acid (g/kg) & $0.02 \pm 0.004$ & $0.62 \pm 0.009$ \\
$\quad$ Lactic acid (g/kg) & $15.74 \pm 0.002$ & $112.33 \pm 0.004$ \\
$\quad$ NEmf (MJ/kg) & $5.68 \pm 0.09$ & $5.76 \pm 0.11$ \\
\hline
\end{tabular}

The values were calculated as the means \pm standard error of the mean $(N=6)$, except for dry matter, others item are based on dry matter 
(3.15, 3.2 and $3.22 \%$, respectively), calcium $(0.43,0.65$ and $0.79 \%$, respectively) and phosphorus $(0.45,0.53$ and $0.48 \%$, respectively).

\section{Temperature and humidity index in the cowshed}

The daily changes in ambient temperature and THI among the three time points is shown in Fig. S1. In general, the THI classification of heat stress is as follows: No heat stress when $\mathrm{THI} \leq 72$; mild heat stress when $72<\mathrm{THI} \leq 79$; high heat stress when $79<\mathrm{THI} \leq 84$; and severe heat stress when THI $>84$ [22]. During the experiment, the mean THI of the barn was 81 (range 79-84), 86 (more than 84), and 79 (range 72-79) in the morning (08:00), afternoon (15:00), and evening (22:00), respectively. The total average THI was 82 , which was between $79<\mathrm{THI}<84$. Therefore, we concluded that fattening cattle were in a high heat stress state during the whole period of the formal experiment.

\section{Effects of fermented HTR on RR and RT of fattening cattle under heat stress}

The daily average $\mathrm{RR}$ of $\mathrm{CN}$ group was 87.04 breaths/ min, and RT was $38.84^{\circ} \mathrm{C}$; RR and RT of LC group were 83.39 breaths $/$ min and $38.75^{\circ} \mathrm{C}$, respectively; and of the $\mathrm{HC}$ group they were 80.23 breaths $/ \mathrm{min}$ and $38.63^{\circ} \mathrm{C}$ (Table 2). At three measurement times, the RR and RT of LC had no significant differences with CN group, while $\mathrm{HC}$ group showed significantly reduced $\mathrm{RR}$ at 15:00 and 22:00 $(P<0.05)$ and a reduced RT at 8:00 and 15:00 $(P<0.05)$ compared with those of the $\mathrm{CN}$ group. At 15:00, the RT of the HC group was significantly lower than that of the LC group $(P<0.05)$. Therefore, $30 \%$ fermented HTR replacement could effectively alleviate the $R R$ and RT of fattening cattle under heat stress.

Table 2 Effects of fermented herbal tea residue feed on respiratory rate and rectal temperature of fattening cattle

\begin{tabular}{lllll}
\hline Items & Time & $\mathbf{C N}$ & LC & HC \\
\hline Respiratory rates & $8: 00$ & $87.12 \pm 1.25$ & $86.67 \pm 1.12$ & $85.00 \pm 1.48$ \\
(breaths/min) & $15: 00$ & $87.50 \pm 2.80^{\mathrm{a}}$ & $83.00 \pm 2.80^{\mathrm{ab}}$ & $78.50 \pm 1.00^{\mathrm{b}}$ \\
& $22: 00$ & $86.50 \pm 3.20^{\mathrm{a}}$ & $80.50 \pm 3.43^{\mathrm{ab}}$ & $77.20 \pm 1.74^{\mathrm{b}}$ \\
Rectal tempera- & $8: 00$ & $38.85 \pm 0.04^{\mathrm{a}}$ & $38.75 \pm 0.04^{\mathrm{ab}}$ & $38.61 \pm 0.05^{\mathrm{b}}$ \\
ture & $15: 00$ & $38.88 \pm 0.06^{\mathrm{a}}$ & $38.78 \pm 0.05^{\mathrm{ab}}$ & $38.60 \pm 0.08^{\mathrm{c}}$ \\
$\left({ }^{\circ} \mathrm{C}\right)$ & $22: 00$ & $38.80 \pm 0.05$ & $38.73 \pm 0.07$ & $38.68 \pm 0.07$ \\
\hline
\end{tabular}

The values were showed as the means \pm standard error $(N=15)$; Different letters showed significant difference $(P<0.05)$, while the same letter or no letter showed no significant difference $(P>0.05)$

HTR herbal tea residue, $C N$ no herbal tea residues, the control group, $L C 15 \%$ fermented HTRs replaced, the $15 \%$ replacement group, $H C$ 30\% fermented HTRs replaced, the $30 \%$ replacement group
Effects of fermented HTR on feed intake and weight gain Compared with the $\mathrm{CN}$ group, the feed intake and daily gain of the $\mathrm{LC}$ and $\mathrm{HC}$ increased gradually, which correlated positively with the increase in fermented HTR replacement (Table 3). The feed intake and average daily gain of the $\mathrm{HC}$ group were significantly higher than those of the $\mathrm{CN}$ group $(P<0.05)$. The average daily gain between the $\mathrm{CN}$ and LC groups showed no significant differences $(P>0.05)$; however, the feed intake increased significantly in the LC group $(P<0.05)$. In addition, fermented HTR also reduced the F/G value of fattening cattle significantly $(P<0.05)$. Therefore, fermented HTR could improve the feed intake and increased the feed conversion rate of fattening cattle under heat stress, and the effect of $30 \%$ fermented HTR replacement was better than that of $15 \%$.

\section{Effects of fermented HTR on serum biochemical indexes}

Compared with the fattening cattle fed with a basal diet, the levels of HSP70 and LDH in the LC and HC groups decreased with the increase in fermented HTR substitution $(P<0.05)$, while IL-2 level increased gradually $(P<0.05)$. Compared with that in the $\mathrm{CN}$ group, the level of Cor in HC group reduced significantly in serum under heat stress $(P<0.05)$, and the levels of IgG and SOD significantly increased $(P<0.05)$. Compared with that in the $\mathrm{CN}$ group, the LDH level in the LC group decreased significantly $(P<0.05)$, and T-AOC level increased significantly in the LC group $(P<0.05)$. IgA, IL-6, ALT, AST, MDA and T4 levels had no significant among three treatments; IgA, IL-6, ALT and AST showed an increased trend, while MDA and T4 showed a decreased trend with the increase of fermented HTR substitution (Table 4). The above results showed that fermented HTR could improve the anti-heat stress and antioxidant capacity of

Table 3 Effect of fermented herbal tea residue feed on feed intake and weight gain of fattening cattle

\begin{tabular}{llll}
\hline Items & CN & LC & HC \\
\hline Initial weight $(\mathrm{kg})$ & $518.29 \pm 27.24$ & $521.43 \pm 38.45$ & $524 \pm 34.21$ \\
Final weight $(\mathrm{kg})$ & $542.94 \pm 26.52$ & $550.13 \pm 37.35$ & $558.33 \pm 32.55$ \\
ADG $(\mathrm{kg} /$ day $)$ & $0.94 \pm 0.05^{\mathrm{b}}$ & $1.09 \pm 0.09^{\mathrm{ab}}$ & $1.26 \pm 0.05^{\mathrm{a}}$ \\
Feed intake $(\mathrm{kg} /$ day) & $12.64 \pm 0.21^{\mathrm{c}}$ & $13.41 \pm 0.21^{\mathrm{b}}$ & $14.27 \pm 0.18^{\mathrm{a}}$ \\
$\begin{array}{l}\text { Feed conversion ratio } \\
\text { (F/G) }\end{array}$ & $13.85 \pm 1.07^{\mathrm{a}}$ & $12.61 \pm 0.53^{\mathrm{b}}$ & $11.64 \pm 0.40^{\mathrm{c}}$ \\
\hline
\end{tabular}

The values were calculated as the means \pm standard error $(N=15)$; Different letters showed significant difference $(P<0.05)$, while the same letter or no letter showed no significant difference $(P>0.05)$

HTR herbal tea residue; $C N$ no herbal tea residues, the control group, $L C 15 \%$ fermented HTRs replaced, the $15 \%$ replacement group, HC 30\% fermented HTRs replaced, the $30 \%$ replacement group 
Table 4 Analysis of serum indices in beef cattle under heat stressed between three groups

\begin{tabular}{llll}
\hline Items & CN & LC & HC \\
\hline HSP70 (ng/mL) & $18.67 \pm 1.17^{\mathrm{a}}$ & $17.78 \pm 0.54^{\mathrm{b}}$ & $16.87 \pm 1.60^{\mathrm{c}}$ \\
Cor $(\mathrm{ng} / \mathrm{mL})$ & $91.55 \pm 1.83^{\mathrm{a}}$ & $80.96 \pm 0.60^{\mathrm{ab}}$ & $72.63 \pm 4.22^{\mathrm{b}}$ \\
$\mathrm{LDH}(\mathrm{U} / \mathrm{L})$ & $696.00 \pm 10.65^{\mathrm{a}}$ & $601.00 \pm 8.97^{\mathrm{b}}$ & $583.00 \pm 3.76^{\mathrm{b}}$ \\
$\mathrm{lgG}(\mathrm{ng} / \mathrm{mL})$ & $7.96 \pm 1.16^{\mathrm{b}}$ & $9.62 \pm 0.44^{\mathrm{b}}$ & $12.30 \pm 1.18^{\mathrm{a}}$ \\
$\operatorname{lgA}(\mathrm{ng} / \mathrm{mL})$ & $3.57 \pm 0.24$ & $4.29 \pm 0.43$ & $4.60 \pm 0.66$ \\
IL-2 $(\mathrm{pg} / \mathrm{mL})$ & $308.75 \pm 2.40^{\mathrm{c}}$ & $332.81 \pm 2.16^{\mathrm{b}}$ & $340.47 \pm 0.43^{\mathrm{a}}$ \\
IL-6 $(\mathrm{pg} / \mathrm{mL})$ & $316.17 \pm 15.25$ & $327.69 \pm 4.43$ & $336.81 \pm 4.80$ \\
ALT $(\mathrm{U} / \mathrm{L})$ & $27.50 \pm 2.02$ & $29.50 \pm 0.87$ & $31.00 \pm 3.21$ \\
AST $(\mathrm{U} / \mathrm{L})$ & $78.00 \pm 6.06$ & $84.50 \pm 9.49$ & $90.25 \pm 4.94$ \\
T-AOC $(\mathrm{mmol} / \mathrm{L})$ & $0.27 \pm 0.03^{\mathrm{b}}$ & $0.36 \pm 0.01^{\mathrm{a}}$ & $0.33 \pm 0.01^{\mathrm{ab}}$ \\
MDA (mmol/L) & $25.21 \pm 1.67$ & $24.93 \pm 0.00$ & $23.47 \pm 0.85$ \\
SOD $(\mathrm{U} / \mathrm{mL})$ & $48.73 \pm 2.79^{\mathrm{b}}$ & $51.76 \pm 0.58^{\mathrm{ab}}$ & $56.43 \pm 0.20^{\mathrm{a}}$ \\
T4 $(\mathrm{ng} / \mathrm{mL})$ & $33.02 \pm 1.06$ & $33.47 \pm 0.36$ & $32.46 \pm 0.70$ \\
\hline
\end{tabular}

The values were showed as the means \pm standard error $(N=6)$; Different letters showed significant difference $(P<0.05)$, while the same letter or no letter showed no significant difference $(P>0.05)$

Cor Cortisol, LDH Lactate dehydrogenase, IgA Immunoglobulin A, IgG Immunoglobulin G, IL-2 Interleukin-2, IL-6 Interleukin-6, ALT Alanine aminotransferase, AST Aspartate aminotransferase, $T$-AOC Total antioxidant capacity, MDA Malondialdehyde, SOD Superoxide dismutase, T4 Thyroxine, HTR Herbal tea residue, $C N$ no herbal tea residues, the control group, $L C 15 \%$ fermented HTRs replaced, the 15\% replacement group, HC 30\% fermented HTRs replaced, the $30 \%$ replacement group

fattening cattle, and the effect of $30 \%$ replacement was more obvious than that of $15 \%$ replacement.

Compared with that in the $\mathrm{CN}$ group, $30 \%$ fermented HTR could reduce blood glucose significantly, and 15 and $30 \%$ fermented HTR replacement could increase the content of total protein and albumin significantly. The content of blood urea nitrogen (BUN), total cholesterol (T-CHO) and triglyceride (TG) had no significant among three treatments; BUN and T-CHO showed a decreased trend with the increase of fermented HTR replacement, while TG showed an increased trend (Table 5).

\section{S rRNA gene sequencing and annotation analysis}

To study the effect of fermented HTR on intestinal microorganisms, we studied the fecal microbiota composition of 6 fattening cattle in control and 30\% HTR replacement groups. We extracted the genomic DNA of 12 fecal samples from $\mathrm{CN}$ group and $\mathrm{HC}$ groups, and amplified the corresponding 16S DNA V3-V4 fragment. After sequencing, the $\mathrm{CN}$ group produced an average of 89,720 raw reads and the $\mathrm{HC}$ group produced an average of 90,069 raw reads. After splicing, the $\mathrm{CN}$ group produced an average of 88,745 combined reads and the average combined percentage was $98.92 \%$. The $\mathrm{HC}$ group produced an average of 88,679 combined reads and the average combined percentage was $98.47 \%$. After filtering low quality and short length fragments, the number of tag
Table 5 Effect of fermented herbal tea residue on biochemical indicators of fattening cattle during summer heat stressed

\begin{tabular}{llll}
\hline Items & CN & LC & HC \\
\hline Glu (mmol/L) & $2.09 \pm 0.008^{\mathrm{a}}$ & $2.06 \pm 0.03^{\mathrm{ab}}$ & $1.99 \pm 0.006^{\mathrm{b}}$ \\
$\mathrm{TP}(\mathrm{g} / \mathrm{L})$ & $67.27 \pm 2.15^{\mathrm{b}}$ & $74.87 \pm 0.67^{\mathrm{a}}$ & $77.25 \pm 1.01^{\mathrm{a}}$ \\
$\mathrm{ALB}(\mathrm{g} / \mathrm{L})$ & $32.23 \pm 0.65^{\mathrm{b}}$ & $33.67 \pm 0.52^{\mathrm{a}}$ & $36.65 \pm 1.88^{\mathrm{a}}$ \\
$\mathrm{BUN}(\mathrm{mmol} / \mathrm{L})$ & $3.35 \pm 0.31$ & $3.04 \pm 0.17610$ & $2.69 \pm 0.07$ \\
$\mathrm{~T}-\mathrm{CHO}(\mathrm{mmol} / \mathrm{L})$ & $2.72 \pm 0.20$ & $2.34 \pm 0.28$ & $2.46 \pm 0.02$ \\
$\mathrm{TG}(\mathrm{mmol} / \mathrm{L})$ & $0.127 \pm 0.03$ & $0.14 \pm 0.01$ & $0.15 \pm 0.009$
\end{tabular}

The values were showed as the means \pm standard error $(N=6)$; Different letters showed significant difference $(P<0.05)$, while the same letter or no letter showed no significant difference $(P>0.05)$

Glu Glucose, ALB Albumin, TPTotal protein, BUN blood urea nitrogen, $T$-CHOTotal cholesterol, TG Triglyceride, HTR Herbal tea residue, $C N$ no herbal tea residues, the control group, LC $15 \%$ fermented HTRs replaced, the $15 \%$ replacement group, HC 30\% fermented HTRs replaced, the $30 \%$ replacement group

sequences for subsequent analysis were 58,016 nochime reads in the $\mathrm{CN}$ group, with an average effective rate of $64.93 \%$, and 58,636 nochime reads in the HC group, with an average effective rate of $65.21 \%$ (Table S3A). The $\mathrm{CN}$ group data generated 1451 OTUs and the HC group generated 1446 OTUs on average (Table S3B). There were 669 core OTUs in the 12 samples (Fig. 1A); with 319 unique OTUs in the $\mathrm{CN}$ group and 297 OTUs in the HC group, while there were 1786 shared OTUs between the two groups (Fig. 1B; Table S3C).

The relative abundance of the top 10 fecal microbiota at the phylum level is shown Fig. $1 \mathrm{C}$ and D. The most abundant bacteria at the phylum level were Firmicutes and Bacteroidetes within and between the $\mathrm{CN}$ and $\mathrm{HC}$ groups. The average percentage of Firmicutes in HC group was $59.54 \%$, which was significantly higher than that in the $\mathrm{CN}$ group $(52.94 \%)(P<0.01)$. The average percentage of Bacteroidetes in the HC group was $31.87 \%$, which was significantly lower than that in the $\mathrm{CN}$ group (37.87\%) $(P<0.01)$. The annotated counts and percentages at the class, order, family, genus, and species levels are show in Table S4 and S5.

Observed species, the Shannon index, the Simpson index, Chao1, ACE, Good's coverage, and PD whole tree reflected the richness and diversity of species in the samples. The species richness of the HC group was slightly higher than that of the $\mathrm{CN}$ group, but there was no significant difference $(P>0.05)$ (Table 6$)$. The PCA between the groups is shown in Fig. 2A. We found that $\mathrm{CN}$ and $\mathrm{HC}$ groups samples were separated from each other, which reflected the influence of HTR substitution on microbial community changes. Similar results were obtained and showed in Fig. 2B. The UPGMA clustering tree was shown in Fig. $2 \mathrm{C}$, which confirmed the significant structural separation of the fecal microflora 

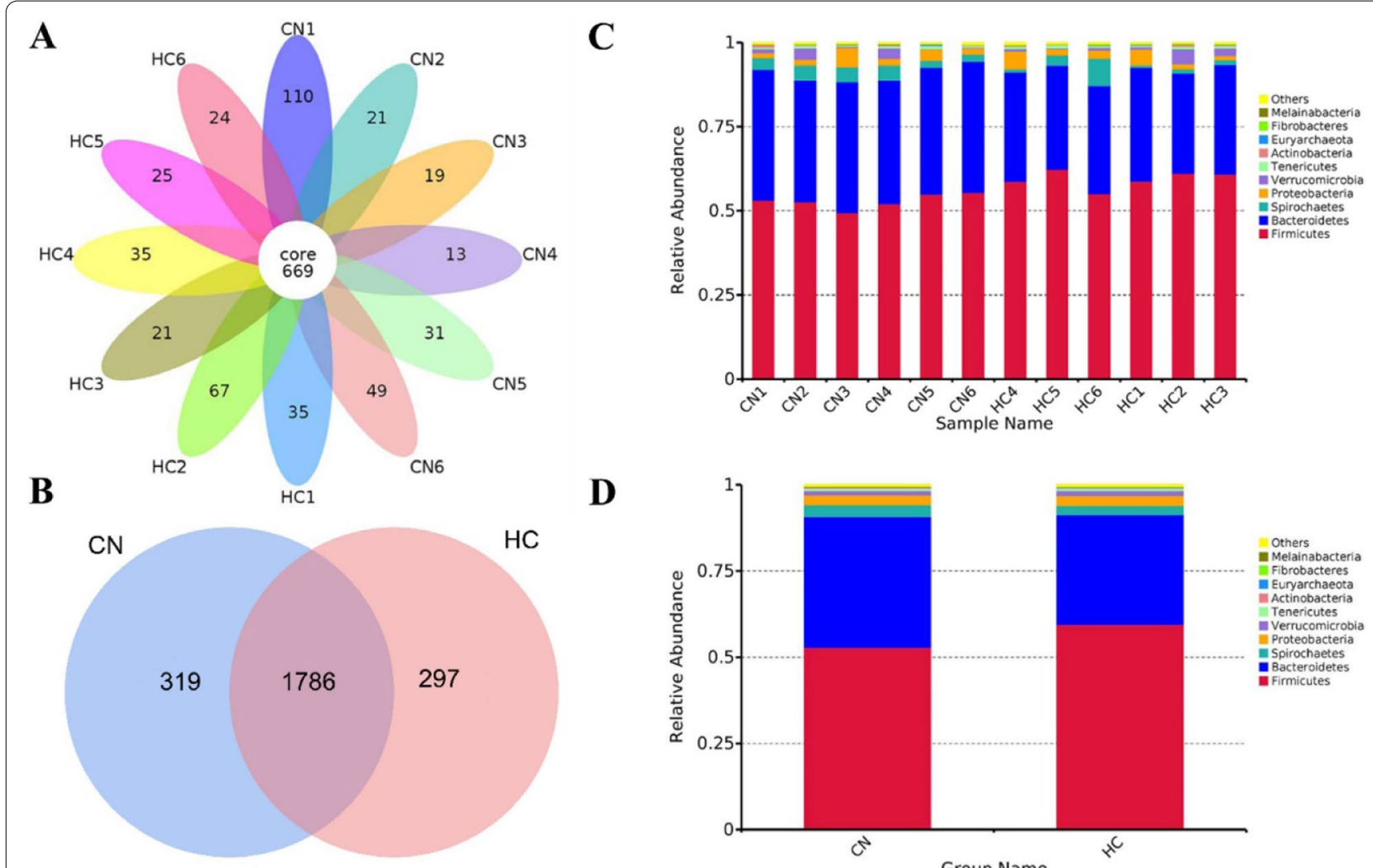

D

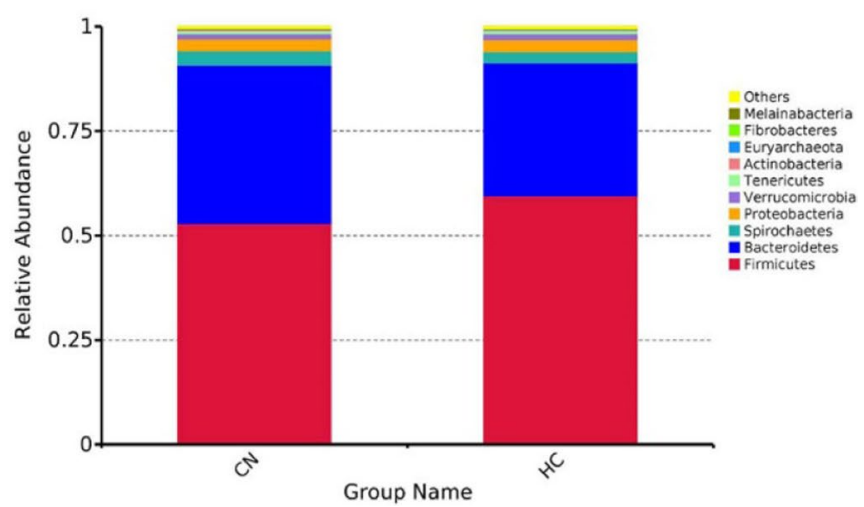

Fig. 1 Common and specific OTU distribution of the fecal microbiota in each sample (A) and two experimental groups (B); relative abundance of fecal microbiota at the phylum level in each sample (C) and in two group (D). Note: OTU, operational taxonomic units; CN, no herbal tea residues, the control group; HC, 30\% fermented HTRs replaced, the 30\% replacement group

Table 6 Statistical analysis of sample complexity

\begin{tabular}{llll}
\hline Item & CN & HC & P vaule \\
\hline Observed species & $1260.50 \pm 15.1805$ & $1265.50 \pm 16.5726$ & 0.828 \\
Shannon & $8.02 \pm 0.0537$ & $8.07 \pm 0.0668$ & 0.564 \\
Simpson & $0.99 \pm 0.0006$ & $0.99 \pm 0.0010$ & 0.568 \\
Chao1 & $1316.69 \pm 16.97$ & $1319.46 \pm 17.83$ & 0.913 \\
Ace & $1321.61 \pm 18.36$ & $1320.44 \pm 17.79$ & 0.964 \\
Goods coverage & $0.99 \pm 0.0001$ & $0.99 \pm 0.0001$ & 0.334 \\
PD whole tree & $85.54 \pm 2.3358$ & $93.59 \pm 2.7638$ & 0.05 \\
\hline
\end{tabular}

Alpha diversity reflected the richness and diversity of species in samples, and it has a variety of indicators: observed species, shannon, simpson, chao1, ace, goods coverage, PD whole tree. The higher the value of Observed species, the higher the species richness are. Shannon index evaluates the richness and evenness of species composition in the sample. The greater the value, the more abundant and evenly distributed the species are. The Chao 1 and ACE indices measure species richness. Goods coverage index represents sequencing depth. PD whole tree index reflects phylogenetic diversity

HTR herbal tea residue, CN no herbal tea residues, the control group, HC 30\% fermented HTRs replaced, the $30 \%$ replacement group between the two groups by measuring the similarity of microbial communities according to the degree of their overlap.

The data in Table S6 show that the levels the Bacteroidia and Clostridia in the $\mathrm{HC}$ group were significantly lower and higher, respectively, than those in the $\mathrm{CN}$ group at the class level $(P<0.01)$, while the Bacteroidales and Clostridiales $(P<0.01)$ showed the same trend at the order level. At the family level, the Rikenellaceae and Paludibacteraceae levels were decreased in the HC group $(P<0.01$ and $P<0.05)$, and the Ruminococcaceae levels were increased $(P<0.05)$. At the genus level, Erysipelotrichaceae levels in the HC group increased $(P<0.01)$, and Fournierella, Acetobacterium, Anaerovorax, Butyrivibrio, and Oscillibacter levels in the $\mathrm{HC}$ group were also higher than those in the $\mathrm{CN}$ group $(P<0.05)$, whereas, the Alistipes levels were lower $(P<0.05)$. At the species level, the rumen_bacterium_NK4A214 were enriched in the HC group $(P<0.05)$. LEFSe identified 29 differentially abundant taxonomic clades in the $\mathrm{CN}$ and $\mathrm{HC}$ groups, whose LDA scores were greater than 2.0 (Fig. 3). The results showed that at the phylum level, the Bacteroidetes 

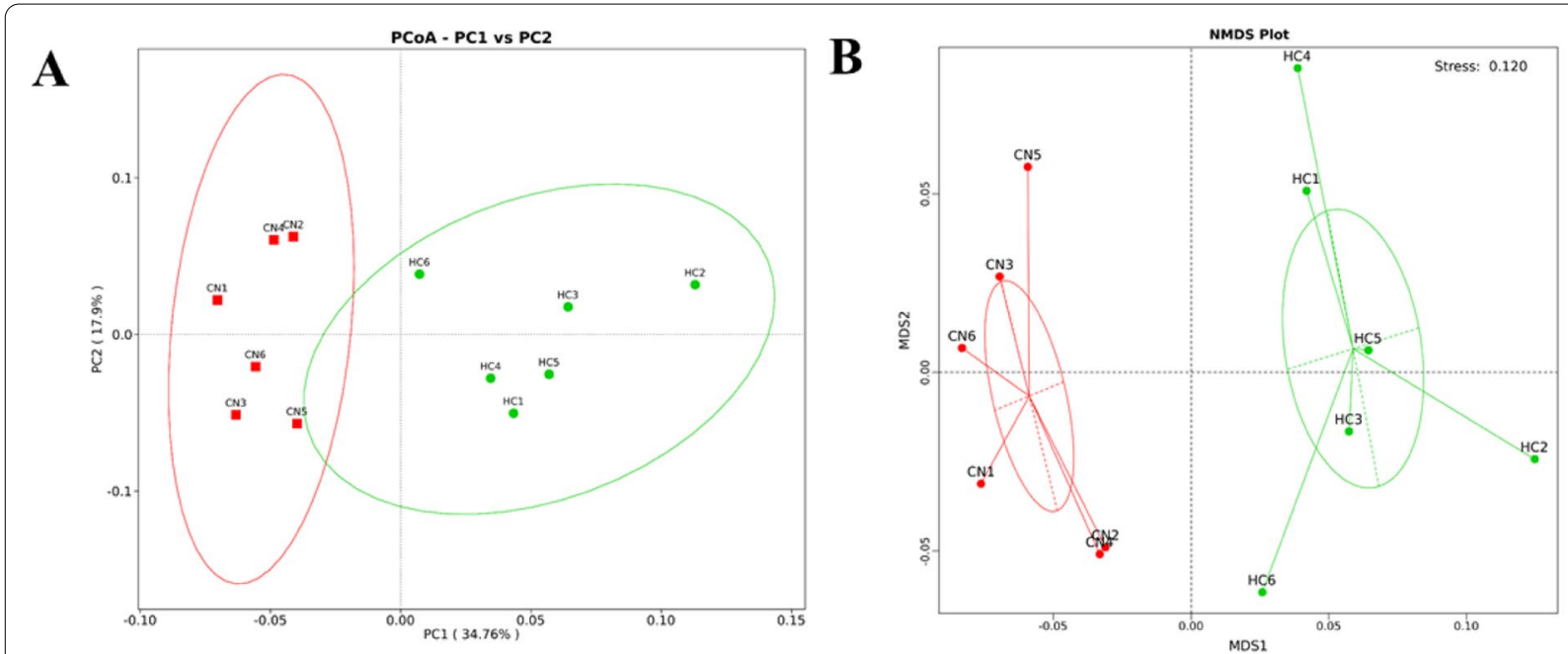

C<smiles>C=CC#N</smiles>

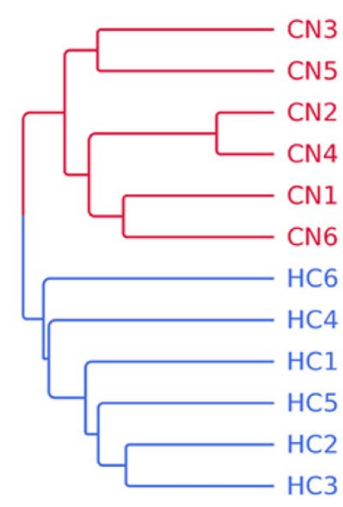

weighted_unifrac Distance

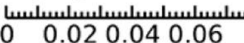

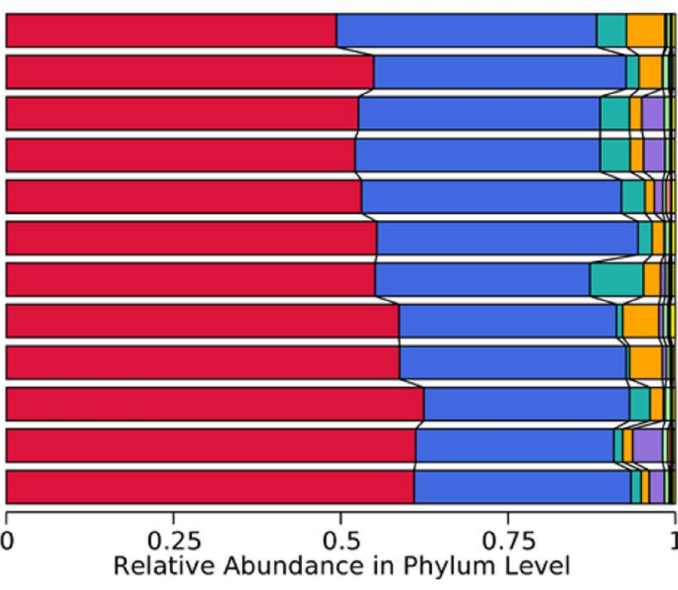

Relative Abundance in Phylum Leve

Fig. 2 Principal component analysis between different groups. Note: PCOA (A), NMDS (B) and UPGMA (C) clustering representing for Principal Co-ordinates Analysis, Non-Metric Multi-Dimensional Scaling and Unweighted Pair-group Method with Arithmetic Mean algorithms, respectively; $\mathrm{CN}$, the control group; $\mathrm{HC}$ is the $30 \%$ replacement group; OTU, operational taxonomic units

were enriched in the $\mathrm{CN}$ group, while the Fibrobacteres and Firmicutes were enriched in the HC group.

\section{Fecal SCFAs concentration and their correlation with microbiota genera}

SCFAs are the main products of microbial metabolism in the intestines, and the types and quantity of SCFAs are regulated by microbial species, diet, and the environment [23]. As shown in Table 7, the SCFAs of the fecal microorganisms in the $\mathrm{CN}, \mathrm{LC}$ and $\mathrm{HC}$ groups showed different trends. The concentration of Aa increased gradually with the increase in fermented HTR substitution, at 13.33, 13.37 , and $14.82 \mathrm{mg} / \mathrm{g}$ in the three groups, respectively. The Aa content in the $\mathrm{HC}$ group was significantly higher than that in the $\mathrm{CN}$ and LC groups. The concentrations of $\mathrm{Pa}(3.29,3.46$, and $3.542 \mathrm{mg} / \mathrm{g}$, respectively), $\mathrm{Ba}(2.35$, 2.51 , and $2.49 \mathrm{mg} / \mathrm{g}$, respectively), and $\mathrm{Va}(0.09,0.09$, and $0.10 \mathrm{mg} / \mathrm{g}$, respectively) increased in the fermented HTR replacement groups, without statistical significance among the groups. The concentrations of Ia were 0.15 , 0.13 , and $0.17 \mathrm{mg} / \mathrm{g}$, respectively, with the concentrations in $\mathrm{HC}$ group being significantly higher than those in the $\mathrm{CN}$ and LC groups. However, the concentration of Iva $(0.22,0.18$, and $0.18 \mathrm{mg} / \mathrm{g}$, respectively) in the $\mathrm{LC}$ and $\mathrm{HC}$ groups were significantly lower than those in the $\mathrm{CN}$ group. We also studied the correlation between SCFAs and significantly enriched fecal microbiota at the genus level. As shown in Fig. 4, there was a significant positive correlation between Aa and the relative abundance of Acetobacterium $(P<0.01)$, and a positive correlation with the relative abundance of Anaerovorax $(P=0.05)$. In addition, there was a positive correlation between $\mathrm{Pa}$ and the relative abundance of Acetobacterium $(P<0.05)$, Erysipelotrichaceae $(P<0.05)$, and Fournierella $(P<0.05)$. 


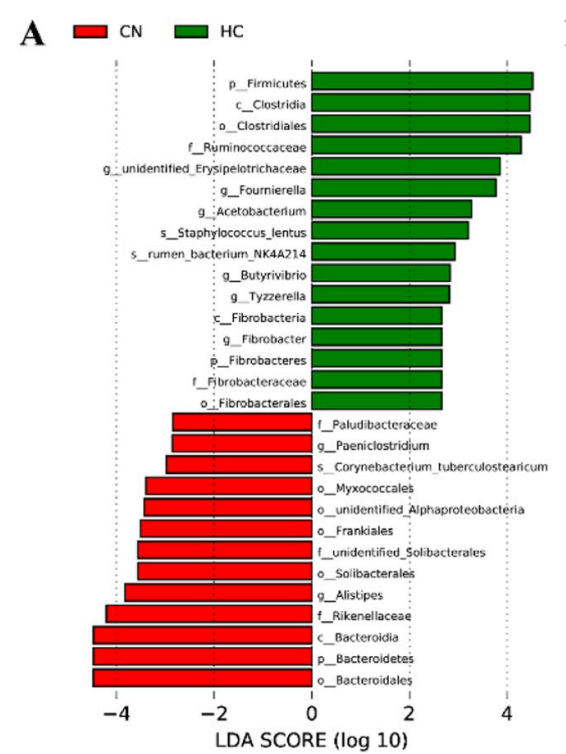

$\mathbf{B}$
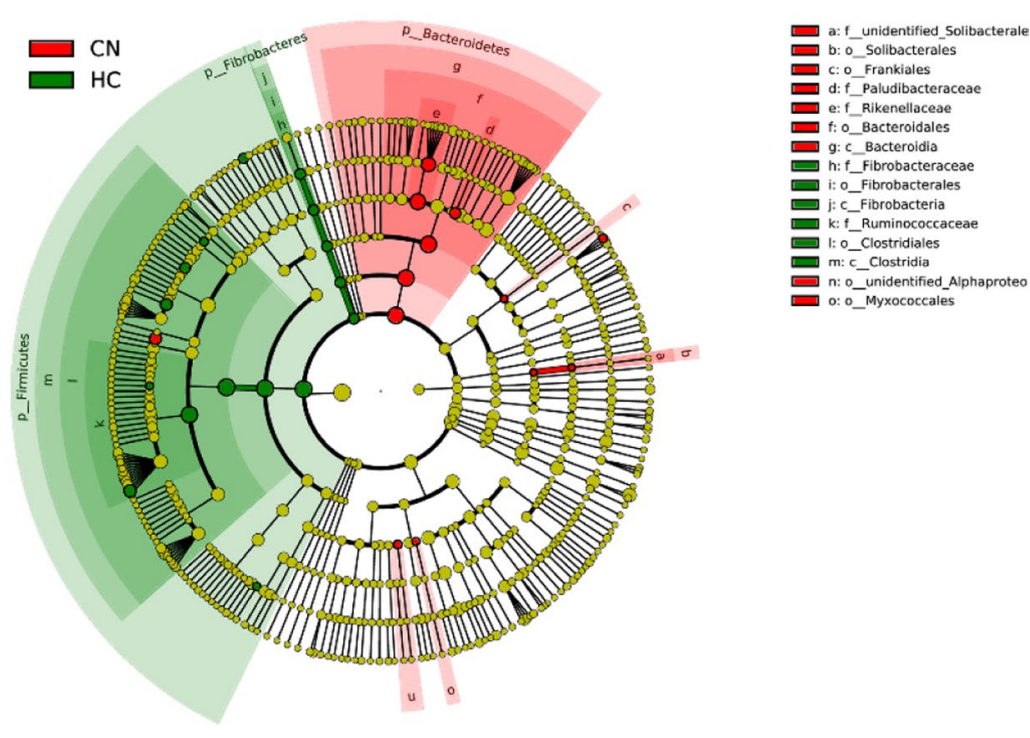

Fig. 3 Taxonomic differences of fecal microbiota between CN and HC groups derived from the LefSe method. Note: A LDA scores observed for individual taxa that passed the LefSe significance threshold of 4. B Taxonomic cladogram. Taxa with enriched levels in CN were showed in red, whereas those with enriched levels in $\mathrm{HC}$ were showed in green. The brightness of the respective colors is proportional to the observed effect size. $\mathrm{CN}$, no herbal tea residues, the control group; HC, 30\% fermented HTRs replaced, the 30\% replacement group

Table 7 Effect of fermented herbal tea residue on the content of short chain fatty acids (SCFAs) in feces of fattening cattle $(\mathrm{mg} / \mathrm{g})$ $(N=6)$

\begin{tabular}{llll}
\hline Item & CN & LC & HC \\
\hline Acetic acid & $13.33 \pm 0.91^{\mathrm{b}}$ & $13.37 \pm 1.27^{\mathrm{b}}$ & $14.82 \pm 0.95^{\mathrm{a}}$ \\
Propionic acid & $3.29 \pm 0.22$ & $3.46 \pm 0.63$ & $3.54 \pm 0.26$ \\
Isobutyric acid & $0.15 \pm 0.01^{\mathrm{b}}$ & $0.13 \pm 0.01^{\mathrm{b}}$ & $0.17 \pm 0.02^{\mathrm{a}}$ \\
Butyric acid & $2.35 \pm 0.16$ & $2.51 \pm 0.22$ & $2.49 \pm 0.21$ \\
Isovaleric acid & $0.22 \pm 0.03^{\mathrm{a}}$ & $0.18 \pm 0.02^{\mathrm{b}}$ & $0.18 \pm 0.02^{\mathrm{b}}$ \\
Valeric acid & $0.09 \pm 0.01$ & $0.09 \pm 0.01$ & $0.10 \pm 0.02$
\end{tabular}

The values were showed as the means \pm standard error $(N=6)$; Different letters showed significant difference $(P<0.05)$, while the same letter or no letter showed no significant difference $(P>0.05)$

$C N$ no herbal tea residues, the control group, LC 15\% fermented HTRs replaced, the $15 \%$ replacement group, HC 30\% fermented HTRs replaced, the $30 \%$ replacement group

However, there was no significant relationship between Ia, Ba, Iva, Va, and Acetobacterium, Alistipes, Anaerovorax, Butyrivibrio, Erysipelotrichaceae, Fournierella, or Oscillibacter $(P>0.05)$.

\section{Discussion}

HTR is the residual water-insoluble substance produced by herbal tea beverage, which has the potential to be used as feed [21]. Like corn silage with a high NDF and Lingnin contents [17], in this experiment, HTR contained more NDF (54.30\% DM) and Lingnin (8.60\%
DM). Higher fiber level can enhance rumen peristalsis, and more NDF can also provide substrate and energy for roughage fermentation in rumen [24], indicating that HTR could be used as a source of ruminant roughage. To improve the palatability of HTR and solve the problem of storage, we fermented the HTR. However, the content of NDF was higher than $54 \%$ and the content of water soluble carbohydrate (WSC) was only $3.10 \%$, which was not conducive to fermentation alone [25]. The addition of molasses increased the WSC of fermented materials [26], and oat hay helped to control the total water content at the beginning of fermentation. In addition, complex bacteria were added to help fermentation. Previous studies have shown that Bacillus subtilis grows strongly and can inhibit the growth of other aerobic and harmful microorganisms during the fermentation process [27]. At the same time, it can produce rich metabolites, such as organic acids and bioactive substances and during fermentation, Bacillus subtilis also produces a large number of enzymes [27]. Yeast is suitable for growth in acidic and humid environments containing sugar. Yeast can use monosaccharides or oligosaccharides produced in fermentation to produce alcohol and carbon dioxide, which is one of the best combination fermentation strains. Lactobacillus can inhibit the growth of spoilage bacteria, reduce the $\mathrm{pH}$, maintain an acidic environment, and ensure the fermentation quality [28]. After fermentation, the content of lactic acid, acetic acid, and propionic acid increased, and the $\mathrm{pH}$ decreased to 3.89 , which effectively 


\begin{tabular}{|c|c|c|c|c|c|c|c|}
\hline Acetobacterium & $\begin{array}{c}0.78 \\
(0.003)\end{array}$ & $\begin{array}{c}0.64 \\
(0.03)\end{array}$ & $\begin{array}{c}-0.076 \\
(0.8)\end{array}$ & $\begin{array}{l}0.42 \\
(0.2)\end{array}$ & $\begin{array}{c}-0.22 \\
(0.5)\end{array}$ & $\begin{array}{l}0.35 \\
(0.3)\end{array}$ & $\Gamma^{1}$ \\
\hline Alistipes & $\begin{array}{l}-0.42 \\
(0.2)\end{array}$ & $\begin{array}{c}-0.057 \\
(0.9)\end{array}$ & $\begin{array}{c}-0.059 \\
(0.9)\end{array}$ & $\begin{array}{l}-0.23 \\
(0.5)\end{array}$ & $\begin{array}{l}0.11 \\
(0.7)\end{array}$ & $\begin{array}{l}0.064 \\
(0.8)\end{array}$ & \multirow{2}{*}{-0.5} \\
\hline Anaerovorax & $\begin{array}{c}0.57 \\
(0.05)\end{array}$ & $\begin{array}{l}0.36 \\
(0.3)\end{array}$ & $\begin{array}{l}0.23 \\
(0.5)\end{array}$ & $\begin{array}{l}-0.2 \\
(0.5)\end{array}$ & $\begin{array}{l}0.081 \\
(0.8)\end{array}$ & $\begin{array}{l}0.43 \\
(0.2)\end{array}$ & \\
\hline Butyrivibrio & $\begin{array}{l}0.27 \\
(0.4)\end{array}$ & $\begin{array}{l}0.45 \\
(0.1)\end{array}$ & $\begin{array}{l}-0.33 \\
(0.3)\end{array}$ & $\begin{array}{l}0.48 \\
(0.1)\end{array}$ & $\begin{array}{l}-0.33 \\
(0.3)\end{array}$ & $\begin{array}{l}-0.19 \\
(0.6)\end{array}$ & -0 \\
\hline Erysipelotrichaceae & $\begin{array}{l}0.49 \\
(0.1)\end{array}$ & $\begin{array}{c}0.63 \\
(0.03)\end{array}$ & $\begin{array}{c}0.014 \\
\text { (1) }\end{array}$ & $\begin{array}{l}0.15 \\
(0.7)\end{array}$ & $\begin{array}{l}-0.11 \\
(0.7)\end{array}$ & $\begin{array}{l}0.35 \\
(0.3)\end{array}$ & \\
\hline Fournierella & $\begin{array}{l}0.47 \\
(0.1)\end{array}$ & $\begin{array}{c}0.67 \\
(0.02)\end{array}$ & $\begin{array}{l}-0.11 \\
(0.7)\end{array}$ & $\begin{array}{c}0.1 \\
(0.8)\end{array}$ & $\begin{array}{l}-0.26 \\
(0.4)\end{array}$ & $\begin{array}{l}0.28 \\
(0.4)\end{array}$ & -0.5 \\
\hline Oscillibacter & $\begin{array}{l}0.038 \\
(0.9)\end{array}$ & $\begin{array}{l}0.38 \\
(0.2)\end{array}$ & $\begin{array}{l}-0.26 \\
(0.4)\end{array}$ & $\begin{array}{c}-0.054 \\
(0.9)\end{array}$ & $\begin{array}{l}-0.27 \\
(0.4)\end{array}$ & $\begin{array}{c}-0.096 \\
(0.8)\end{array}$ & \\
\hline
\end{tabular}

Fig. 4 Statistical analysis of correlation between genus taxa and fecal SCFA. Note: Each cell contained the corresponding correlation and $P$ value. The table is color-coded by correlation according to the color legend (Red indicates positive correlation and green indicates negative correlation)

inhibited the reproduction of undesirable bacteria, increased the content of crude protein and decreased the content of NDF, indicating a better fermentation quality. Previous study found that green tea residue silage could be used as a protein source for lactating dairy cows to replace part of the alfalfa and soybean meal in their diet, which had no adverse effect on the performance of the dairy cows [29], suggesting that fermented HTR could be used as feed resource.

In the subtropical region of southern China, the climate is hot in summer; therefore, beef cattle are vulnerable to summer heat stress, which affects their eating and health [30]. This results in growth retardation, which affects the development of the beef cattle industry [31]. It has been reported that heat stress can reduce the intake of feed and affect the metabolism after absorption [32]. The temperature and humidity index reflects the heat stress intensity of animals by measuring the temperature and humidity index of the breeding environment [33]. Our results showed that the total average THI was 82 , which was between $79<\mathrm{THI}<84$. Therefore, we concluded that the fattening cattle were in a high heat stress state during the whole period of the formal experiment. RR and RT are indices used to quantify the physiological changes of animals under heat stress [34]. Replacement with fermented HTR was beneficial to reduce the RT and RR of fattening cattle in the heat stress environment, with the $30 \%$ fermented HTR having a more significant effect. This suggested that fermented HTR could alleviate heat stress symptoms of fattening cattle in summer, which is consistent with the results of other studies [35-37]. Chen found that Chinese herbal medicine could reduce significantly the RR of beef cattle under heat stress [36]. Song found that Chinese herbal medicine could reduce the RT for a certain period of time under high temperature [35].
When animals are exposed to heat stress, they eat less to reduce their heat production [37], which ultimately affects their growth and fattening.

Cor is a hormone secreted by the adrenal gland during stress. When the body is in the state of heat stress, it will stimulate the adrenal cortex to secrete Cor to adapt to the adverse environment [38]. Our results showed that in the heat stress environment, with the increase in fermented HTR in the diet, the amount of Cor secreted by the fattening cattle decreased gradually, indicating that fermented HTR could effectively alleviate the heat stress response of fattening cattle. This was consistent with the results of Song et al., who found that Chinese herbal medicine could reduce the secretion of Cor in beef cattle under heat stress [35] and this might be explained by the fact that herbal medicine active substances are retained in the HTR, which can relieve heat stress in summer. LDH participates in glycolysis. Hocking et al. found that high heat treatment can increase LDH activity [39]. In our results, LDH level decreased with the increase of fermented HTR replacement, indicating that fermented HTR could alleviate heat stress in fattening cattle.

When the animals are in the state of heat stress for a long time, the body's immunity will decline [40]. IgG is the most abundant immunoglobulin in animal organs, which can protect the body from infection, and is an important immune index under heat stress [41]. Interleukin-2 (IL-2) is a cell growth factor in the immune system of the body, and is also an important indicator of cellular immune function under heat stress [42]. Furthermore, IL-6 is a proinflammatory cytokine that is associated with an increased inflammatory response [43]. Studies have shown that HTR can enhance the immune function of the human body [44]. In the present study, we found that the levels of IgG and IL-2 
in the serum of beef cattle fed with fermented HTR increased significantly, which indicated that fermented HTR could enhance the immune function of beef cattle under heat stress.

The content of MDA indirectly reflects the degree of lipid peroxidation in tissues and cells, and can be used as a surrogate for the degree of cell damage [45]. SOD is an antioxidant enzyme that can scavenge free radicals in vivo. Studies show that heat stress can inhibit the expression of SOD [46]. T-AOC represents the antioxidant capacity of the whole body. In this study, the MDA level had a decreased trend with the increase of fermented HTR replacement, and we therefore inferred that fermented HTR may be required to alleviate the cell damage caused by heat stress. T-AOC level increased significantly in 15\% substitution and SOD level increased significantly in 30\% substitution group, reflecting the antioxidant activity of fermented HTR, which was consistent with Chaudhary's findings [47].

In addition, some studies have suggested that to adapt to heat stress, the body needs to increase the absorption of glucose to ensure the energy supply, resulting in an increased serum glucose content [48]. In the present study, there was no significant difference in the total cholesterol level with increasing fermented HTR. However, under heat stress, the glucose level of the fattening cattle fed with the basal diet was significantly higher than that of cattle fed with the $30 \%$ fermented HTR alternative diet, suggesting that heat stress has an effect on the glucose level, which can be alleviated by fermented HTR. Compared with those of the $\mathrm{CN}$ group, the total protein and albumin of the fermented HTR groups increased while blood urea nitrogen showed a decreased trend, which was similar with Guo's results [49]. Guo pointed out that this might be the result of thyroid regulation, which was conducive to maintaining the body's water and salt balance, and alleviating the adverse effects of heat stress [49].

At present, there are few studies on the effect of HTR on the fecal microorganisms of fattening cattle under heat stress. Recently, a study showed that there are significant differences in the microbial composition between spring and summer, indicating that environmental temperature has an impact on the microbial community structure of feces [50]. In the present study, we found that $30 \%$ fermented HTR had a more significant effect on heat stress index of fattening cattle than 15\%; therefore, we chose the $\mathrm{CN}$ and $\mathrm{HC}$ group to study the fecal microbial community of fattening cattle. According to the results of alpha diversity analysis and PCA, the fecal microbial community of heat stressed fattening cattle fed with fermented HTR was significantly different from cattle fed with the basic diet, and the microbial diversity of the $\mathrm{HC}$ group increased. The most abundant bacteria in the phylum level of the $\mathrm{CN}$ and $\mathrm{HC}$ group were Firmicutes and Bacteroidetes. Li showed that under the different climates in spring and summer, the main microorganisms in cow feces were Firmicutes and Bacteroidetes; in summer, Firmicutes levels were higher than those in spring, while Bacteroidetes levels were lower than those in spring [50]. It has been reported that ruminants have a core microbial community mainly composed of Firmicutes and Bacteroidetes, which can maintain stable digestive function [51]. In our study, the feeding of fermented HTR changed the relative proportion of these two bacteria, which was consistent with Hu's study [52]. The results showed that the enrichment of Firmicutes in the HC group was significantly higher than that in $\mathrm{CN}$ group, and Bacteroidetes levels in $\mathrm{HC}$ group were significantly lower, indicating that fermented HTR had a certain effect on fecal microbial flora of fattening cattle under summer heat stress. It is reported that Bacteroidetes is mainly responsible for the degradation of fiber, and its degree of enrichment is related to diets with a high fiber content. By contrast, Firmicutes are responsible for the degradation of carbohydrates, fats, and proteins, which are related to a high calorie diet [53]. We infer that the increase of Firmicutes in $\mathrm{HC}$ group is related to the richer crude protein and crude fat in fermented HTR.

At the family taxonomic level, the Rikenellaceae and Paludibacteraceae in the HC group decreased, and the Ruminococcaceae increased. It has been reported that the Rikenellaceae increased and the Ruminococcaceae decreased in the intestines of mice fed with a high-fat diet, suggesting that a high calorie diet can cause changes in the intestinal microflora [54], which is contrary to the results of the $\mathrm{HC}$ group and might be explained by the fact that under heat stress, the fermented HTRs reduced the caloric level of the diet, alleviated the heat production during fattening, and changed the intestinal flora. At the genus taxonomic level, Fournierella and Oscillibacter are two genera of the Ruminococcaceae, and both showed an increasing trend in the HC group. Acetobacterium, Anaerovorax, Butyrivibrio, and Erysipelotrichaceae also increased in the HC group. A study has reported that Butyrivibrio is more involved in epithelium promotion, while Erysipelotrichaceae is more abundant at high temperatures [55]. Besides, Alistipes decreased in the HC group. A study suggested that Alistipes is the source of colorectal cancer, and its abundance is related to the severity of intestinal diseases [56], which indicates that fermented HTR is beneficial to maintain the health of fattening cattle under heat stress.

We also studied the association between fecal SCFAs and microorganisms at the genus level where changes were observed. We found that there was a significant 
positive correlation between $\mathrm{Aa}$ and the relative abundance of Acetobacterium and a positive correlation between $\mathrm{Pa}$ and the relative abundance of Acetobacterium, Erysipelotrichaceae, and Fournierella. It has been reported that Acetobacter is mainly involved in Aa fermentation [57] and Fournierella can metabolize and produce $\mathrm{Aa}$ and $\mathrm{Pa}$ [58]. SCFAs are the main products of microbial metabolism in the intestines, and the types and quantity of SCFAs are regulated by microbial species, diet, and the environment [23]. Therefore, we speculated that fermented HTRs change the composition and abundance of large intestine microorganisms in fattening cattle, promote the fermentation of large intestine microorganisms, and help the body to maintain health and resist the adverse effects of heat stress.

\section{Conclusions}

After fermentation with oat hay and compound bacteria, the $\mathrm{pH}$ of the HTR decreased and the nutritional level increased, which was conducive to the resource utilization of HTR. In addition, fermented HTR could alleviate the heat stress of fattening cattle in summer, improve their growth performance, enhance their immune performance, help them to resist an adverse environment, and maintain their health. In addition, 30\% fermented HTR replacement had an impact on the fecal microbial community of heat stressed fattening cattle, and the bacteria were closely related to the cattle's fecal SCFA levels. Based on our findings, we concluded that $30 \%$ fermented HTRs replacing Pennisetum purpureum grass might be advantageous for fattening cattle under heat stress. In addition, the co-fermented products also included molasses, oat hay, and mixed bacterial strains in our test, and the potential roles of these co-fermented products on heat stress should be further study.

\section{Materials and methods}

\section{Fermentation}

The HTRs used in the experiment were obtained from Heyuan Jilongxiang Biological Technology Co., LTD. (Heyuan, Guangdong, China). The molasses were purchased from Guangzhou Linong Feed Co., Ltd. (Guangzhou, Guangdong, China) and strains were purchased from Guangdong Microbial Culture Collection Center (Guangzhou, Guangdong, China). The feedstock department of Guangzhou Fengxing Dairy Co., LTD. (Guangzhou, Guangdong, China) provided the oat hay.

Based on dry matter, the tested HTR contained 20\% Mesona chinensis leaves, 20\% Plumeria rubra flowers, $20 \%$ Microcos paniculata leaves, $12 \%$ Honeysuckle flowers, $12 \%$ Chrysanthemum flowers, $12 \%$ Prunella vulgaris leaves and $4 \%$ Liquiritia glycyrrhiza roots in mass ratios, respectively. The nutritional components of HTR was analyzed by E-more Technology Co., Ltd. (Ulanqab, Inner Mongolia, China). In details, HTR was grind to power in 1 mil with UDY-cyclone (UDY Corporation, Fort Collins, CO); then scanned by NIR spectroscopy with 'Feed and Forage' system (1100-2500 nm) (Thermo Scientific, Waltham, Massachusetts, US) and merged with Dairy on (https://dairyone.com/) database.

In this experiment, HTR and oat hay were cut into $2-3 \mathrm{~cm}$ pieces. Fermentation was carried out on HTR and oat hay (CP: $12.3 \%$; NDF: $50.8 \%$; ADF: $26.7 \%$ ) with a mass ratio of $6: 4$ on a wet weight basis (approximately $3: 7$ on dry matter basis), with $2 \%$ added molasses. The mixed bacteria used for fermentation were Saccharomyces cerevisiae GDMCC2.167 $\left(3 \times 10^{8}\right.$ colony forming units/g), Bacillus subtilis GDMCC1.372 $\left(3 \times 10^{8}\right.$ colony forming units/g), Lactobacillus plantarum GIM1.191 ( $5 \times 10^{9}$ colony forming units/g) and Enterococcus faecalis GDMCC1.612 $\left(3 \times 10^{8}\right.$ colony forming units/g), with mass ratios of $250 \mathrm{~g}: 1 \mathrm{t}, 300 \mathrm{~g}: 1 \mathrm{t}, 300 \mathrm{~g}: 1 \mathrm{t}$, and $200 \mathrm{~g}: 1 \mathrm{t}$, respectively. The fermentation materials were mixed and stirred evenly using a feed-stuff mixer. Then the mixture was compacted to remove air and pressed into polyethylene bags $(50 \mathrm{~kg}$ each) and sealed, and fermented for 25-30 days at room temperature. After approximately 30 days of fermentation, $20 \mathrm{~g}$ of fermented samples were added to $180 \mathrm{~mL}$ of distilled water, sealed with preservative film, and placed in a refrigerator at $4^{\circ} \mathrm{C}$ for $24 \mathrm{~h}$. During extraction, the solution was shaken every $30 \mathrm{~min}$. After $24 \mathrm{~h}$, the solution was filtered through qualitative filter paper, and a pH meter (Horiba, Tokyo, Japan) was used to determine the $\mathrm{pH}$ value of the filtrate. High performance liquid chromatography (HPLC) was used to determine the content of organic acids in the extract of fermented HTRs, while AOAC International guidelines [59] were used to analyze the dry matter, crude protein, $\mathrm{NDF}, \mathrm{ADF}$, and ash contents.

\section{Experimental animals and design}

This experiment was carried out in Guangzhou from July to August in 2019, and the weather is hot, rainy and humid, which usually leads to a natural heat stress and we judged whether the individuals were in the state of heat stress by using the environmental temperature and humidity index [22]. A total of 45 healthy female Simmental crossbred cattle (18 months old), balanced with body weight, were selected and divided into three treatment groups (15 cattle per group) in the same cowshed: The CN group (fed with basal diet, without fermented HTR), the LC group (fermented HTR replaced 15\% of Pennisetum purpureum grass), and the HC group (fermented HTR replaced $30 \%$ of Pennisetum purpureum grass). The experiment lasted for 40 days, including a 
7-day adaptation period and a 33-day formal experimental period.

The animal feeding experiment was carried out at the Yunfu benben beef cattle farm (Yunfu, Guangdong, China), and the basal diet for the experimental cattle was provided by the farm and was shown in Table S2. During the experiment, all individuals were fed at 8:00 am and 5:00 pm every day. A total mixed ration (TMR) feed mixer was used to fully mix the experimental diets, and the diet and drinking water were allowed to intake freely during the experiment.

\section{Measurements and sampling}

During the experiment, a dry and wet thermometer (Kimo Industry Co., Biarritz, France) was set at $1.5 \mathrm{~m}$ above the center of ground in the cowshed. The temperature, humidity, and wet bulb temperature of the cowshed were recorded at 08:00, 14:00, and 20:00 every day to calculate the daily average temperature, humidity and the temperature humidity index (THI) of the cowshed. The following formula was used to calculate the THI: $\mathrm{THI}=$ $(0.35 \times \mathrm{TDB}+0.65 \times \mathrm{TWB}) \times 1.8+32$, where $\mathrm{TDB}$ is the dry bulb temperature and TWB is the wet bulb temperature [22].

In every 3 days, an animal thermometer (Kruuse, Langeskov, Denmark) was used to measure the rectal temperature (RT) of the experimental cattle at 08:00, 14:00, and 22:00 during the formal experimental period. When the tested cattle lay still, we counted the times of chest fluctuation in $1 \mathrm{~min}$ with the counter, which was recorded as respiratory rate (RR). At the beginning and end of the formal experiment, the weights of the experimental cattle were recorded after a 12-h overnight fast. The amount of TMR consumed by the experimental cattle, and that remaining unconsumed, were recorded every day. The above data were used to calculate the average daily feed intake (ADFI), average daily gain (ADG), and the feed to weight ratio (F/G). On the day before the end of the experiment, six individuals in each treatment group were randomly selected and $20 \mathrm{~mL}$ blood samples were taken from the tail vein, placed in a centrifuge tube, and then centrifuged immediately after being placed obliquely for $2 \mathrm{~h}$. Enzyme linked immunosorbent assay (ELISA) kits were used to determine the serum levels of heat shock protein 70 (HSP70), cortisol (Cor), lactate dehydrogenase (LDH), immunoglobulin G (IgG), immunoglobulin A (IgA), alanine aminotransferase (ALT), creatine kinase (CK), total antioxidant capacity (T-AOC), malondialdehyde (MDA), superoxide dismutase (SOD), glutathione peroxidase (GSH-PX) according to the manufacturer instrutions (Nanjing Jiancheng Bioengineering Research Institute, Nanjing, China).
At the end of the experiment, six animals of each group were randomly selected and $200 \mathrm{~g}$ feces were collected from 12 fattening cattle rectums in $\mathrm{CN}$ and $\mathrm{HC}$ groups, then put into a frozen storage tube and stored in liquid nitrogen. Approximately $100 \mathrm{~g}$ were used for HPLC (Actlabs, Ancaster, ON, Canada) determination of fecal volatile fatty acid contents, including acetic acid (Aa), propionic acid $(\mathrm{Pa})$, isobutyric acid $(\mathrm{Ia})$, butyric acid $(\mathrm{Ba})$, isovaleric acid (Iva), and valeric acid (Va). The other $100 \mathrm{~g}$ were used to extract total genomic DNA to investigate fecal microorganisms and the total genomic DNA was extracted using CTAB/SDS method. DNA concentration and purity was monitored on $1 \%$ agarose gels. According to the concentration, DNA was diluted to $1 \mathrm{ng} / \mu \mathrm{L}$ using sterile water.

\section{S rRNA gene sequencing and annotation analysis}

We amplified the V3 and V4 regions of the 16S rRNA sequence of the bovine fecal microbial communities by using a forward primer (5'-GTGCCAGCMGCCGCG G-3') and a reverse primer (5'-GGACTACHVGGGTWT CTAAT $-3^{\prime}$ ), and then constructed gene fragment libraries. The paired end method was used to sequence the libraries on the Illumina Novaseq sequencing platform (Illumina, Waltham, MA, USA). High-quality tags data (clean tags) were obtained after splicing and filtering of the reads [60], and then mosaic filtering was performed to obtain effective tags for subsequent analysis. The Uparse software (Uparse v7.0.1001) [61] was used to cluster all effective tags of all samples. Sequences with $\geq 97 \%$ identity were clustered into same operational taxonomic units (OTUs). Then, Silva databases and Mothur [62] were used to annnotate and analyze the OTU sequences. The composition and abundance of microbial communities in each sample were counted at each taxonomic level, including kingdom, phylum, class, order, family, genus, and species. Fast multiple sequence alignment and annotation was carried out using the MUSCLE software (Version 3.8.31), and the phylogenetic relationships between different OTUs were obtained [63]. Finally, the data of each sample were homogenized, and the subsequent Alpha and Beta diversity were analyzed based on the homogenized data. The QIIME pipeline (Version 1.7.0) was used to calculate the UniFrac distance and an Unweighted Pair-group Method with Arithmetic Means (UPGMA) clustering tree was also constructed. The R software (Version 2.15.3) was used to draw Principal Component Analysis (PCA), Principal Co-ordinates Analysis (PCoA), and Non-Metric Multi-Dimensional Scaling (NMDS) maps to explore the differences of community structure among the different samples. The Linear discriminant analysis Effect Size (LEfSe) analysis was performed with a linear discriminant analysis LDA score of 2 to further analyze the differences in community structure. 


\section{Statistical analysis}

One-way analysis of variance (ANOVA) and Duncan's test (SPSS 17.0, IBM Corp., Armonk, NY, USA) were used to analyze the physiological parameters, serum biochemical indices, and the fecal concentrations of short-chain fatty acids (SCFAs). The results are shown as the mean \pm standard error (SE), and $P<0.05$ and $P<0.01$ were used as the criteria to judge significant and extremely significant differences.

\section{Supplementary Information}

The online version contains supplementary material available at https://doi. org/10.1186/s12917-021-03061-y.

Additional file 1 : Figure S1. Daily changes in cowshed ambient temperature and humidity index $(\mathrm{THI})$ in three time. The red line represents the daily changes of $\mathrm{THI}$ index at 8:00 am, the orange line represents the daily changes of THI index at 15:00 pm, and the gray line represents the daily changes of THI index at 22:00 pm. Supplementary Tables S1-S6.

\section{Authors' contributions}

$X Z$ and JS conceived the report, participated in its design, performed data analysis, and drafted the manuscript; ZC, XS and FL performed in experiments. JL, TC, QX and YZ provided guidance and funding. All authors read and approved the final manuscript.

\section{Funding}

This work was supported by the Natural Science Foundation of China Program [grant numbers 31802032, 32072714]; the Natural Science Foundation of Guangdong Province [grant number 2020A1515010062]; the Science and Technology Project of Guangzhou [grant number 202002030037]; and the Key-Area Research and Development Program of Guangdong Province [2019B110209005].

This work was supported by the Natural Science Foundation of China Program [grant numbers 31802032, 32072714]; the Natural Science Foundation of Guangdong Province [grant number 2020A1515010062]; the Science and Technology Project of Guangzhou [grant number 202002030037]; and the Key-Area Research and Development Program of Guangdong Province [2019B110209005].

\section{Availability of data and materials}

The raw sequences were deposited into Sequence Read Archive (SRA) database with the BioProject accession number PRJNA699165.

\section{Declarations}

\section{Ethics approval and consent to participate}

The animal feeding experiment was carried out at the Yunfu benben beef cattle farm (Yunfu, Guangdong, China). The tested animals were housed in an open cowshed, and animals were fed individually. All animal procedures were approved by the owner of farm and the Animal Care Committee at South China Agricultural University according to the university's guidelines for animal research (2018F006).

\section{Consent for publication}

Not applicable.

\section{Competing interests}

The authors declare that they have no competing interests.

Received: 28 August 2021 Accepted: 31 October 2021 Published online: 12 November 2021

\section{References}

1. Li FS, Weng JK. Demystifying traditional herbal medicine with modern approach. Nat Plants. 2017;3:17109.

2. Chio PH, Zaroff CM. Traditional Chinese medicinal herbal tea consumption, self-reported somatization, and alexithymia. Asia Pac Psychiatry. 2015;7(2):127-34.

3. Desideri D, Meli MA, Roselli C, Feduzi L. Alpha and gamma spectrometry for determination of natural and artificial radionuclides in tea, herbal tea and camomile marketed in Italy. Microchem J. 2011;98(1):170-5.

4. Zhao J, Deng JW, Chen YW, Li SP. Advanced phytochemical analysis of herbal tea in China. J Chromatogr A. 2013;1313:2-23.

5. Dalar A, Konczak I. Phenolic contents, antioxidant capacities and inhibitory activities against key metabolic syndrome relevant enzymes of herbal teas from eastern anatolia. Ind Crop Prod. 2013;44:383-90.

6. Shen J, Shan J, Zhu X, Xu Y, Li M. Effect of Chinese herbal tea on health tested on drosophila model. Plant Foods Hum Nutr. 2020;75(2):305-6.

7. Yang D, Liang J, Wang Y, Sun F, Tao H, Xu Q, et al. Tea waste: an effective and economic substrate for oyster mushroom cultivation. J Sci Food Agric. 2010;96(2):680-4.

8. Kondo M, Kita K, Yokota H. Effects of tea leaf waste of green tea, oolong tea, and black tea addition on sudangrass silage quality and in vitro gas production. J Sci Food Agric. 2004;84(7):721-7.

9. Ozdemir N, Yakupoglu T, Dengiz O. The effects of bio-solid and tea waste application into different levels of eroded soil on N, P and K concentrations. Environ Monit Assess. 2009;156(1-4):109-18.

10. Hameed BH. Spent tea leaves: a new non-conventional and low-cost adsorbent for removal of basic dye from aqueous solutions. J Hazard Mater. 2009;161(2-3):753-9.

11. Shang JG, Kong XR, He LL, Li WH, Liao QJH. Low-cost biochar derived from herbal residue: characterization and application for ciprofloxacin adsorption. Int J Environ Sci Technol. 2016;13(10):2449-58.

12. Ahsan MA, Katla SK, Islam MT, Hernandez-Viezcas JA, Martinez LM, DíazMoreno CA, et al. Adsorptive removal of methylene blue, tetracycline and $\mathrm{Cr}(\mathrm{VI})$ from water using sulfonated tea waste. Environ Technol Innov. 2018;11:23-40.

13. Cheraghi M, Sobhanardakani S, Lorestani B, Zandipak R. Tea wastes efficiency on removal of cd(ii) from aqueous solutions. Arch Hyg Sci. 2016;5(3):184-91.

14. Dizadji N, Anaraki NA. Adsorption of chromium and copper in aqueous solutions using tea residue. Int J Environ Sci and Tech. 2011;8(3):631-8.

15. Wan S, Qu N, He F, Wang M, Liu G, He H. Tea waste-supported hydrated manganese dioxide (hmo) for enhanced removal of typical toxic metal ions from water. RSC Adv. 2015;5:88900-7.

16. Lunge S, Singh S, Sinha A. Magnetic iron oxide (fe3o4) nanoparticles from tea waste for arsenic removal. J Magn Magn Mater. 2014;356:21-31.

17. Hristov AN, Harper MT, Roth G, Canale C, Huhtanen P, Richard TL, et al. Effects of ensiling time on corn silage neutral detergent fiber degradability and relationship between laboratory fiber analyses and in vivo digestibility. J Dairy Sci. 2020;103(3):2333-46.

18. Liu HW, Tong JM, Zhou DW. Utilization of chinese herbal feed additives in animal production. Agric Sci China. 2011;10:1262-72.

19. Samanta AK, Jayaram C, Jayapal N, Sondhi N, Kolte AP, Senani S, et al. Assessment of fecal microflora changes in pigs supplemented with herbal residue and prebiotic. PLoS One. 2015;10(7):e0132961.

20. Liu W, Rouzmehr F, Seidavi A. Effect of amount and duration of waste green tea powder on the growth performance, carcass characteristics, blood parameters, and lipid metabolites of growing broilers. Environ Sci Pollut Res Int. 2018;25(1):375-87.

21. Xie Y, Chen Z, Wang D, Chen G, Sun X, He Q, et al. Effects of fermented herbal tea residues on the intestinal microbiota characteristics of Holstein heifers under heat stress. Front Microbiol. 2020;11:1014.

22. Berman A. Estimates of heat stress relief needs for Holstein dairy cows. J Anim Sci. 2005;83(6):1377-84.

23. Macfarlane S, Macfarlane GT. Regulation of short-chain fatty acid production. Proc Nutr Soc. 2003;62(1):67-72.

24. Zou Y, Zou X, Li X, Guo G, Ji P, Wang Y, et al. Substituting oat hay or maize silage for portion of alfalfa hay affects growth performance, ruminal fermentation, and nutrient digestibility of weaned calves. Asian-Australas J Anim Sci. 2018;31(3):369-78.

25. Smith LH. Theoretical carbohydrate requirement for alfalfa silage production. Agron J. 1962;54(4):291-3. 
26. Thompson DN, Barnes JM, Houghton TP. Effect of additions on ensiling and microbial community of senesced wheat straw. Appl Biochem Biotechnol. 2005;121-124:21-46.

27. Chen KL, Kho WL, You SH, Yeh RH, Tang SW, Hsieh CW. Effects of Bacillus subtilis var. natto and Saccharomyces cerevisiae mixed fermented feed on the enhanced growth performance of broilers. Poult Sci. 2009;88(2):309-15.

28. Wang C, He L, Xing Y, Zhou W, Yang F, Chen X, et al. Fermentation quality and microbial community of alfalfa and stylo silage mixed with Moringa oleifera leaves. Bioresour Technol. 2019;284:240-7.

29. Kondo M, Nakano M, Kaneko A, Agata H, Kita K, Yokota H. Ensiled green tea waste as partial replacement for soybean meal and alfalfa hay in lactating cows. Asian-Aust J Anim Sci. 2004;17(7):960-6.

30. Hahn GL. Dynamic responses of cattle to thermal heat loads. J Anim Sci. 1999;77(Suppl 2):10-20.

31. St-Pierre NR, Cobanov B, Schnitkey G. Economic losses from heat stress by us livestock industries 1. J Dairy Sci. 2003;86:52-77.

32. Belhadj Slimen I, Najar T, Ghram A, Abdrrabba M. Heat stress effects on livestock: molecular, cellular and metabolic aspects, a review. J Anim Physiol Anim Nutr (Berl). 2016;100(3):401-12.

33. Zhong S, Ding Y, Wang Y, Zhou G, Guo H, Chen Y, et al. Temperature and humidity index (THI)-induced rumen bacterial community changes in goats. Appl Microbiol Biotechnol. 2019;103(7):3193-203.

34. da Costa AN, Feitosa JV, Montezuma PA Jr, de Souza PT, de Araújo AA. Rectal temperatures, respiratory rates, production, and reproduction performances of crossbred Girolando cows under heat stress in northeastern Brazil. Int J Biometeorol. 2015:59(11):1647-53.

35. Song X, Luo J, Fu D, Zhao X, Bunlue K, Xu Z, et al. Traditional chinese medicine prescriptions enhance growth performance of heat stressed beef cattle by relieving heat stress responses and increasing apparent nutrient digestibility. Asian-Australas J Anim Sci. 2014;27(10):1513-20.

36. Chen J, Guo K, Song X, Lan L, Liu S, Hu R, et al. The anti-heat stress effects of Chinese herbal medicine prescriptions and rumen-protected $\gamma$-aminobutyric acid on growth performance, apparent nutrient digestibility, and health status in beef cattle. Anim Sci J. 2020;91(1):e13361.

37. Wang J, Li J, Wang F, Xiao J, Wang Y, Yang H, et al. Heat stress on calves and heifers: a review. J Anim Sci Biotechnol. 2020;11:79.

38. Chanclón B, Martínez-Fuentes AJ, Gracia-Navarro F. Role of SST, CORT and ghrelin and its receptors at the endocrine pancreas. Front Endocrinol (Lausanne). 2012;3:114.

39. Hocking PM, Maxwell MH, Mitchell MA. Haematology and blood composition at two ambient temperatures in genetically fat and lean adult broiler breeder females fed ad libitum or restricted throughout life. $\mathrm{Br}$ Poult Sci. 1994:35(5):799-807.

40. Dahl GE, Tao S, Laporta J. Heat stress impacts immune status in cows across the life cycle. Front Vet Sci. 2020;7:116

41. Liu DY, He SJ, Liu SQ, Tang YG, Jin EH, Chen HL, et al. Daidzein enhances immune function in late lactation cows under heat stress. Anim Sci J. 2014;85(1):85-9.

42. Padgett DA, Glaser R. How stress influences the immune response. Trends Immunol. 2003;24(8):444-8.

43. Esposito G, Irons PC, Webb EC, Chapwanya A. Interactions between negative energy balance, metabolic diseases, uterine health and immune response in transition dairy cows. Anim Reprod Sci. 2014;144(3-4):60-71.

44. Costa LB, Luciano FB, Miyada VS, Gois FD. Herbal extracts and organic acids as natural feed additives in pig diets. S Afr J Anim Sci. 2013;43:181-93.

45. Tsikas D. Assessment of lipid peroxidation by measuring malondialdehyde (MDA) and relatives in biological samples: analytical and biological challenges. Anal Biochem. 2017;524:13-30.
46. Yang CY, Lin MT. Oxidative stress in rats with heatstroke-induced cerebral ischemia. Stroke. 2002;33(3):790-4.

47. Chaudhary SS, Singh VK, Upadhyay RC, Puri G, Odedara AB, Patel PA. Evaluation of physiological and biochemical responses in different seasons in Surti buffaloes. Vet World. 2015;8(6):727-31.

48. Sun $X$, Zhang H, Sheikhahmadi A, Wang Y, Jiao H, Lin H, et al. Effects of heat stress on the gene expression of nutrient transporters in the jejunum of broiler chickens (Gallus gallus domesticus). Int J Biometeorol. 2015:59(2):127-35.

49. Guo K, Cao H, Zhu Y, Wang T, Hu G, Kornmatitsuk B, et al. Improving effects of dietary rumen protected $\gamma$-aminobutyric acid additive on apparent nutrient digestibility, growth performance and health status in heat-stressed beef cattle. Anim Sci J. 2018;89(9):1280-6.

50. Li H, Li R, Chen H, Gao J, Wang Y, Zhang Y, et al. Effect of different seasons (spring vs summer) on the microbiota diversity in the feces of dairy cows. Int J Biometeorol. 2020;64(3):345-54.

51. Weimer PJ. Redundancy, resilience, and host specificity of the ruminal microbiota: implications for engineering improved ruminal fermentations. Front Microbiol. 2015;6:296.

52. Hu X, Liu G, Li Y, Wei Y, Lin S, Liu S, et al. High-throughput analysis reveals seasonal variation of the gut microbiota composition within Forest musk deer (Moschus berezovskii). Front Microbiol. 2018;9:1674

53. Jami E, Israel A, Kotser A, Mizrahi I. Exploring the bovine rumen bacterial community from birth to adulthood. ISME J. 2013;7(6):1069-79.

54. Daniel H, Gholami AM, Berry D, Desmarchelier C, Hahne H, Loh G, et al. High-fat diet alters gut microbiota physiology in mice. ISME J. 2014:8(2):295-308.

55. Ziętak M, Kovatcheva-Datchary P, Markiewicz LH, Ståhlman M, Kozak LP, Bäckhed F. Altered microbiota contributes to reduced diet-induced obesity upon cold exposure. Cell Metab. 2016;23(6):1216-23.

56. Parker BJ, Wearsch PA, Veloo ACM, Rodriguez-Palacios A. The genus Alistipes: gut bacteria with emerging implications to inflammation, cancer, and mental health. Front Immunol. 2020;11:906.

57. Kremp F, Poehlein A, Daniel R, Müller V. Methanol metabolism in the acetogenic bacterium Acetobacterium woodii. Environ Microbiol. 2018;20(12):4369-84.

58. Togo AH, Durand G, Khelaifia S, Armstrong N, Robert C, Cadoret F, et al. Fournierella massiliensis gen. nov., sp. nov., a new human-associated member of the family Ruminococcaceae. Int J Syst Evol Microbiol. 2017;67(5):1393-9.

59. Horwitz W. Official methods of analysis of AOAC International. Volume I, agricultural chemicals, contaminants, drugs/edited by William Horwitz. Gaithersburg: AOAC International; 2020.

60. Bokulich NA, Subramanian S, Faith JJ, Gevers D, Gordon JI, Knight R, et al. Quality-filtering vastly improves diversity estimates from Illumina amplicon sequencing. Nat Methods. 2013;10(1):57-9.

61. Edgar RC. UPARSE: highly accurate OTU sequences from microbial amplicon reads. Nat Methods. 2013;10(10):996-8.

62. Quast C, Pruesse E, Yilmaz P, Gerken J, Schweer T, Yarza P, et al. The SILVA ribosomal RNA gene database project: improved data processing and web-based tools. Nucleic Acids Res. 2013:41(Database issue):D590-6.

63. Edgar RC. MUSCLE: multiple sequence alignment with high accuracy and high throughput. Nucleic Acids Res. 2004;32(5):1792-7.

\section{Publisher's Note}

Springer Nature remains neutral with regard to jurisdictional claims in published maps and institutional affiliations. 